\title{
OSCILLATING SOLUTIONS OF THIRD ORDER DIFFERENTIAL EQUATIONS
}

\author{
W. R. UTZ
}

ABSTRACr. Third order ordinary linear differential equations are investigated with respect to the property of having a basis for solutions that consist of three oscillating solutions yet some nontrivial solution of the equation is nonoscillatory.

1. Introduction. We will consider the equation

$$
y^{\prime \prime \prime}+P(t) y^{\prime \prime}+Q(t) y^{\prime}+R(t) y=0
$$

wherein we will always assume that the coefficients are continuous for all large $t$. A solution of (1) will be called oscillatory if it changes sign for arbitrarily large $t$. All other solutions are said to be nonoscillatory. Clearly, (1) always has the trivial nonoscillatory solution $y \equiv 0$. Much of the oscillation literature for third order equations is cited by C. A. Swanson [6].

It is easy to exhibit an equation (1), even with constant coefficients, for which no solution oscillates. The purpose of the present paper is to go toward the other end of the spectrum by considering equations (1) for which there are three linearly independent oscillating soluions but for which there is a nontrivial nonoscillatory solution.

As an example of such an equation consider $y^{\prime \prime \prime}+y=0$. If $u_{1}=e^{-t}$, $u_{2}=e^{t / 2} \cos (\sqrt{ } 3 t / 2), u_{3}=e^{t / 2} \sin (\sqrt{ } 3 t / 2)$ then the equation has the three linearly independent solutions $u_{1}+u_{2}, u_{2}, u_{3}$ which all oscillate yet, of course, the equation has nontrivial nonoscillatory solutions. By contrast, one can show that it is not possible to find three linearly independent oscillatory solutions of $y^{\prime \prime \prime}-y=0$. The existence of equations (1) for which all nontrivial solutions oscillate has recently been demonstrated by J. M. Dolan [2]. Such equations are not our primary concern here.

If $y_{1}(t), y_{2}(t), y_{3}(t)$ are three class $C^{3}$ functions defined for all large $t$ and if their Wronskian does not vanish for all large $t$ then one can write an equation having these three functions as linearly independent solutions by forming

Received by the editors January 1, 1970 and, in revised form, March 16, 1970.

AMS 1969 subject classifications. Primary 3420; Secondary 3442.

Key words and phrases. Differential equations, third order, oscillation, basis of solutions, selfadjoint equations. 


$$
\left|\begin{array}{llll}
y_{1} & y_{2} & y_{3} & y \\
y_{1}^{\prime} & y_{2}^{\prime} & y_{3}^{\prime} & y^{\prime} \\
y_{1}{ }^{\prime \prime} & y_{2}^{\prime \prime} & y_{3}^{\prime \prime} & y^{\prime \prime} \\
y_{1}{ }^{\prime \prime \prime} & y_{2}{ }^{\prime \prime \prime} & y_{3}{ }^{\prime \prime \prime} & y^{\prime \prime \prime}
\end{array}\right|=0 .
$$

This equation has no singularities (for large $t$ ).

Thus, one can find an equation for which all solutions (not identically zero) oscillate if one can find three oscillating functions $y_{1}, y_{2}, y_{3}$ with nonvanishing Wronskian and such that any linear combination of $y_{1}, y_{2}, y_{3}$ is oscillatory. In fact, this is equivalent to the problem of finding such an equation since if $y_{1}, y_{2}, y_{3}$ are three class $C^{3}$ functions with nonvanishing Wronskian, then these three functions satisfy a unique equation (1).

It is clear that the property of linear combinations of oscillatory functions being oscillatory is vital in the question of the existence of equations (1) for which all nontrivial solutions oscillate. This approach is not unique, however, and, in particular, is not the approach used by Dolan [2].

The equation (2) with solutions $y_{1}=e^{t} \sin t, y_{2}=e^{t} \cos t, y_{3}=e^{t}$ has a basis for all solutions with $0,1,2$ or 3 oscillatory elements. This contrast with the constant coefficient case seems sufficiently novel to warrant status as a theorem. ${ }^{1}$

THEOREM 1. There are examples of equations (1) for which there is a basis for all solutions with $0,1,2,3$ nonoscillatory solutions.

2. In the Introduction we observed that the solutions of $y^{\prime \prime \prime}+y=0$ have a basis in oscillatory functions. We now give a theorem from which this follows as a corollary. In the proof of the theorem we will need the fact that if $u_{1}, u_{2}, u_{3}$ are linearly independent solutions of (1), then so are $u_{1}, u_{1}+u_{3}, u_{2}+u_{3}$. Indeed, they have the same Wronskian. As before, we assume the coefficients $Q(t), R(t)$ to be continuous and now, even more, that $R(t) \in C^{2}$. There is no loss in generality in taking $P(t) \equiv 0$ in (1) for oscillation theorems since this term can be eliminated from (1) by the transformation

$$
y=w \exp \left(-\frac{1}{3} \int_{0}^{t} P(s) d s\right)
$$

\section{THEOREM 2. If in}

${ }^{1}$ The author's argument to support Theorem 1 has been considerably shortened by the argument given here by the referee. 


$$
y^{\prime \prime \prime}+Q(t) y^{\prime}+R(t) y=0
$$

$Q(t) \leqq 0, R(t)>0$,

$$
2 Q(t) / R(t)+d^{2}\left(R^{-1}(t)\right) / d t^{2} \leqq 0
$$

and some solution of (3) oscillates, then there are three linearly independent oscillatory solutions of (3) yet some nontrivial solution is nonoscillatory.

Proof. According to Theorem 1.1 of Lazer [3] the equation has a nontrivial nonoscillating solution, $N(t)$, for which $N(t) \rightarrow c$ as $t \rightarrow \infty$. Unless $Q(t) \equiv 0$ we are unable to be sure that $c=0$ (this interesting open question is discussed by Lazer [3, p. 444]). By Theorem 1.4 of [3] we have two linearly independent oscillatory solutions $u(t), v(t)$ which, with $N(t)$, form a basis for the solutions of (3). Moreover, the absolute values of the extrema of $u(t), v(t)$ are bounded below by $m>0\left[3\right.$, Lemma 1.4]. We now take the nonoscillating solution $N^{*}(t)$ of (3) defined as $N(t)$ if $c=0$, as $(m / 2 c) N(t)$ is $c \neq 0$. With this choice of $N^{*}(t)$, the solutions $u(t)+N^{*}(t), v(t)+N^{*}(t)$ both oscillate and with $u(t)$ (or $v(t)$ ) form a basis for (3) to complete the proof.

In case $Q(t) \equiv 0$ one knows [5] that there is an oscillating solution and, moreover, $c=0$. In this case one has the following corollary to the above theorem.

COROLlary. If in

$$
y^{\prime \prime \prime}+R(t) y=0,
$$

$R(t) \in C^{2}, R(t)>0$ and $d^{2} R^{-1}(t) / d t^{2} \leqq 0$, then there are three linearly independent oscillatory solutions of (4) yet some nontrivial solution does not oscillate.

It is known [1] that all solutions of the selfadjoint equation

$$
u^{\prime \prime \prime}+b(t) u^{\prime}+\frac{1}{2} b^{\prime}(t) u=0
$$

are given by

$$
u=k_{1} y_{1}^{2}+k_{2} y_{1} y_{2}+k_{3} y_{2}^{2}
$$

where $y_{1}, y_{2}$ are linearly independent solutions of

$$
y^{\prime \prime}+\frac{1}{4} b(t) y=0 \text {. }
$$

It is evident that (6) may be nonconstant and nonoscillatory (for example, by taking $\left.b=1, k_{1}=1, k_{2}=k_{3}=0\right)$. Even worse, (5) is known [1] to have a solution without a zero (as is obvious if $b(t)$ is constant). 
The nonoscillation criteria of Lazer do not apply in the case of (5) since he specifically excludes this equation in [3].

Equation (5) may have all solutions periodic even when $b(t)$ is not constant. To secure such equations one may take $[4$, p. 353] a class of continuous functions $b(t)$ for which there are linearly independent periodic (and oscillatory) solutions $y_{1}, y_{2}$ of (7) having a common period. For such functions $b(t)$ all solutions (6) are periodic. We summarize this paragraph in the following theorem.

THEOREM 3. There exist nonconstant functions $b(t)$ for which all solutions of (5) are periodic. However, some solutions are nonoscillatory.

\section{REFERENCES}

1. G. D. Birkoff, On the solutions of ordinary linear homogeneous differential equations of the third order, Ann. of Math. 12 (1911), 103-127.

2. J. M. Dolan, On the relationship between the oscillatory behavior of a linear thirdorder equation and its adjoint, J. Differential Equations 7 (1970), 367-388.

3. A. C. Lazer, The behavior of solutions of the differential equation $y^{\prime \prime \prime}+p(x) y^{\prime}$ $+q(x) y=0$, Pacific J. Math. 17 (1966), 435-466. MR 33 \#1552.

4. C. M. Petty and J. E. Barry, A geometrical approach to the second-order linear differential equation, Canad. J. Math. 14 (1962), 349-358. MR 26 \#3973.

5. M. Švec, Sur une propriêté des intégrales de l'équation $y^{(n)}+Q(x) y=0, n=3,4$, Czechoslovak Math. J. 7 (82) (1957), 450-462. MR 20 \#1816.

6. C. A. Swanson, Comparison and oscillation theory of linear differential equations, Academic Press, New York, 1968.

University of Missouri, Columbia, Missouri 65201 